\title{
Concept of coherence of learning physical optics
}

Elisa Colombo, Mirta Jaen, Leonor de Cudmani

Elisa M. Colombo, Mirta Jaen, Leonor C. de Cudmani, "Concept of coherence of learning physical optics," Proc. SPIE 2525, 1995 International Conference on Education in Optics, (13 October 1995); doi: 10.1117/12.224066

EPIE Event: SPIE's 1995 International Symposium on Optical Science, Engineering, and Instrumentation, 1995, San Diego, CA, United States 
The concept of coherence of learning physical optics

\author{
Elisa Colombo - Mirta Jaen - Leonor C. de Cudmani \\ Instituto de Luminotecnia, Luz y Visión "Ing. Herberto C. Bühler" \\ Instituto de Física \\ Facultad de Ciencias Exactas y Tecnología \\ Universidad Nacional de Tucumán \\ Av. Independencia 1800 - (4000) Tucumán - Argentina
}

\begin{abstract}
The aim of the actual paper is to enhance achievements of the text "Optica Física Básica: estructurada alrededor del concepto de coherencia luminosa" (1) (in English "Basic Physical Optics centred in the concept of coherence"). We consider that this book is a very worth tool when one has to learn or to teach some fundamental concepts of physical optics.

It is well known that the topics of physical optics present not easy understanding for students. Even more they also present some difficulties for the teachers when they have to introduce them to the class.

First, we think that different phenomena like diffraction and polarization could be well understood if the starting point is a deep comprehension of the concept of interference of light and, associated with this, the fundamental and nothing intuitive concept of coherence of the light. In the reference text the authors propose the use of expression "stable interference pattern of no uniform intensity" instead of "pattern of interference" and "average pattern of uniform intensity" instead of "lack of interference" to make reference that light always interfere but just under restrictive conditions it can be got temporal and spatial stability of the pattern.

Another idea we want to stand out is that the ability to observe a "stable interference pattern of no uniform intensity" is associated not only with the coherence of the source but also with the dimensions of the experimental system and with the temporal and spatial characteristics of the detector used - human eye, photographic film, etc.-

The proposal is well support by quantitative relations. With an alternate model: a train of waves with a finite length of coherence, it is possible to get range of validity of models, to decide when a source could be considered a "point" or "monochromatic" or "remote", an "infinite" wave or a train of waves, etc. Using this concept it is possible to achieve a better understanding of phenomena like the polarization of light. Here, it is easier to recognize limitations of the model of light. For example, in the interpretation of the effect of retarding plates on polarizated light. When the plate is wider than the coherence length of the wavetrain of light, the effect disappears.
\end{abstract}

(1) Cudmani, Leonor C. de et al.

Optica Física Básica: Estructurada alrededor del concepto de coherencia luminosa 
Publication Nro. 1441 - National University of Tucumán - Argentina (April 1990)

Keywords: coherence - interference - diffraction - polarization - physical optics - teaching and learning

\section{INTRODUCTION}

It is well known that the topics of physical optics present not easy understanding for students because they involve non intuitive concepts. Even more, they also present some difficulty for the teachers when they have to introduce these concepts to a class. The teaching experience collected from many years shows that there are errors and confusions very rooted difficulting the comprehension of interference, diffraction and polarization phenomena. The most important cores of difficulties that we have detected in our research work about these topics are related with:

* Previous notions are not present. In fact, it is a big difference with the intuitive notion of the students connected with the Mechanics topics.

* Different phenomena involve in the physical optics are studied as isolated compartments. It is no usual to find out the necessary integration between interference, diffraction and polarization.

* There is a deep internalization of a very simplified model of light and it is not clear the range of validity of it. Most of the texts, usually employed to study the Physical Optics, represent the light as an infinite spatial and temporal wave, linearly polarized, with constant amplitude and frequency and determined initial phase. This preconception about the simplified model of light is created in a high proportion by the instruction. Then, it makes more difficult the conceptual change to a new model that permit a deep comprehension of the fundamental concept of coherence of light.

The aim of this paper is to help the students and teachers to get an integration between the mentioned phenomena. The fundamental points that we want to rescue are the following:

* We think that the essential topics of the physical optics could be well understood if the starting point is a deep comprehension of the concept of interference of light and, associated with this, the fundamental and non intuitive concept of coherence of the light.

* It is important to take as starting point all simple phenomena from everyday experience that most of the time is overlooked. For instance, nature exploits the wave properties of light to produce some purest and most striking colours around, in birds, rocks, and across the sky. The students can see interference fringes using the fingers in front of their eyes or a diffraction pattern through a drop of rain on a windscreen. 
* It is useful do the experiment and encourages direct observations at the laboratory facing the students to interactive work. With low cost experiments the students can develop an interesting introduction to the subject that allow the conceptual change between the successive paradigms of the Optics.

* It is necessary to keep the conceptual rigours before manipulations of formulas. In this sense concepts must be well explained and their limits and range of validity have to be explicited. This is the only way to avoid mechanic applications of mathematical expressions or calculations without physical bases.

In this sense, this paper enhances achievements of the text "Basic Physical Optics centred in the concept of coherence"(1). We consider that this book is a very worth tool when we have to learn or to teach some subjects of physical optics.

\section{FORMULATION OF HYPOTHESIS}

Learning optics, it is necessary to select a model according to the relations between the object sizes and the wavelength of the light. Often, the students used each model like a truth but not as a hypothesis that explains the reality. They are not aware about modelling. This is one distinctive characteristic of the scientific knowledge and this is almost overlooked by the traditional education.

Geometrical optics works when the relevant dimensions of the objects that interact with light (lenses, mirrors, barriers, etc.) are much larger than light's wavelength. But, during the instruction it is necessary to introduce more and more complex models of light. The wave model, first the mechanic approach and then the electromagnetic model allows a more comprehensive model to represent the reality. Thus, wave optics is not confined to small or faint corrections of geometrical optics. But even this powerful of the wave model of the light does not give the capacity for to say "light is a wave", expression that you can find also in a physics text. This kind of expression is the basis of most of rooted bad conceptualization in Physics, forgetting the role of the modelling and the connection between model and reality. This fact has serious consequence: Physics appears like a rigid, complete and close science that losses attractive to the students.

Besides the aspects just mentioned, different educational researches $(1,2,3)$ pointed out some reasons that could explain the mistakes and confusions very settled in the study of interference phenomena, difficulting the comprehension of physical phenomena involved. In fact, in this paper, we hypothesize that there are two fundamental reasons:

* The gap between two different models: the infinite electromagnetic wave and the finite wavetrain representing the light. Most of the texts usually employed to study the 
Physical Optics, represent the light as an infinite spatial and temporal wave, linearly polarized, with constant amplitude and frequency and determined initial phase. Even a laser has not characteristics like this ideal wave. We think there are a strong internalization and permanence of this simplified model of light and it is not clear the range of validity of it.

* The wrong association of the coherence concept only with the characteristics of the light sources. Most of the time the concept of coherence is related just with the emitting source, considering the sun or an incandescent lamp, as incoherence sources and a laser as a coherence source, without pay attention to the experimental system dimensions and the detector characteristics. Obviously when more coherent is the light source less important are the restrictions for the dimensions of the device or less critical are the characteristics of the detector. If the wavelength is infinite any system could detect stable patterns of interference. But, if we consider the more realistic representation of light like a finite train of waves, the spatial and temporal sensitivity of the detector play an important role in the observation of the results of the interference.

The mentioned simplification failed when the students consider really experimental system. It is possible to see Young' interference fringes using the sun as a source, and miss interference fringes with two independent lasers. It is interesting that the students can discuss questions like "why we cannot see interference patterns on the lit walls of the laboratory and we can see them in the Young 's experiment using the same no-coherent source"? Then, an essentially no-coherent source in terms of emission, could be considered like a coherent one (plane wavefront) being far from the experimental system. Consequently, it forms a stable pattern of non uniform intensity.

\section{HOW TO UNDERSTAND INTERFERENCE AND COHERENCE}

The two concepts that we consider are the clues to understand these topics in an introductory stage are interference and coherence of the light.

If we have two similar sources with two different amplitudes and we make the observation in a certain direction the resultant energy that we received is proportional to the sum of the two effects when they are alone plus a correction. This correction term is used to call "interference effect". If the interference term is positive, texts refer as "constructive interference" and in the opposite case as "destructive interference". That is, the combined intensity of light shining on something from two sources is not such a sum of the intensities of the two lights because there is also "some interference between them". If there are circumstances in which this term has no importance, texts use to say that the interference is apparently lost. Of course, in nature it is always there, but we may not be able to detect it.

Our eye already has a hole in it that is so large that we are averaging the effects over a range very wide compared with one wavelength. So if we average over regions where the phase of 
the light wave varies very rapidly with position, we get no interference pattern. The same thing happens when the phase difference between two sources is gradually drifting with time, but if our observation is so crude that we cannot see that little time, if these changes are too rapid for our equipment to follow, then again the phase shift averages out and we get no "interference pattern".

Because of this reason, we propose to change the language in a way to express clearly the meaning of interference. Instead of talking about "interference patterns", it would be better uses "stable patterns of dark and brightness" when the sensor is "able" to differentiate, in time and in space, changes of intensity. Simultaneously, instead of "absence of interference", talk about "a uniform intensity pattern", when the sensor "does not detect" the changes.

On other hand, texts usually say: "most of the ordinary light sources change their phase so rapidly and randomly that we cannot observe interference between light from such two sources. Such sources are called incoherent sources. In the same way, sources that stay in step with each other are coherent. Interference can only be seen with coherent beams". Therefore, to observe the wave nature of light we need coherent sources, but where do we find them? The trick is to use one ordinary, monochromatic, point light source and somehow split its light into two beams ( amplitude splitting, wavefront splitting, etc.). Then, however the phase of the source changes, both beams change their phases in step and thus keep up their phase relationship constant. If the path followed by the two beams are not too different, we can recombine them and see their interference.

This shows that in reality, the calling "coherent sources" are not necessary to obtain "stable patterns of dark and brightness"and that the coherence is a property of the all system, including its geometric dimensions and the particular detector used. But this is not always explicit and frequently texts generalize the definitions out of the context, without take account its limitations.

Now, if one looks for a definition of diffraction is common to find that it is an effect produced when a beam of light, narrow enough, passes through a small hole and it spreads out. But it is not always clear that diffraction is explained as interference effects. As Feynman said: " No one has ever been able to define the difference between interference and diffraction satisfactorily. It is just a question of usage, and there is no specific, important physical difference between them... when there are only a few sources, say two, interfering, then the result is usually called interference, but if there is a large number of them, it seems that the word diffraction is more often used ...".

Finally, one believes that to understand the phenomena with polarizated light, the fundamental point is to introduce the electromagnetic vectorial model of light, without pay attention to the times of polarization of the wavetrain and the interference effects involve. Two polarized waves in perpendicular planes can of course interfere, but we are not be able to see any modulated pattern. Instead of that, the polarization state will change as a 
result of this interference. But if we remember that one atom emits one polarized wavetrain during $10^{-8} \mathrm{sec}$, and then another atom emits light with a different polarization. The resultant polarization will change every $10^{-8} \mathrm{sec}$. If the polarization changes more rapidly that we can detect it, texts talk about "unpolarized light", because all the effects of the polarization average out. When we use a polarizer in front of a beam of this light, it selects all the wavetrains polarized in one direction and absorb the others. All the colours effects with polarized light are results of interference effects but we are able to see them only if the length of the wavetrain is enough to produce interference between two parts of the same train. The clue of these experimental systems is that the wave perturbations that interfere have their origin in the same, never in two independent wavetrains. For this reason if the retard plate is wider than the length of coherence we will not see colours effects and none of the interference effects of polarization would show up with "unpolarized light".

\section{4.- CONCLUSION: SIMPLE QUANTITATIVE CRITERIA}

We want to stand out that the proposal is well supported by quantitative relations. With the alternate model consisting of a train of wave with a finite length of coherence, is possible to get the limits of validity of models. It is possible to decide when a source could be considered a "point-source" or a "monochomatic" or a "remote" source, a wavetrain "infinite". Even more, using this concept, it is easier to recognize limitations of the model, for example, in the interpretation of the effect of retarding plates on polarized light. When the plate is wider than the coherence length of the wavetrain of the light, the effect disappears.

First, it is important to take in account the orders of magnitude involved in the experiment: the finite spatial and temporal durations of the luminous waves train, the reaction time of the detector. If we focalized our attention in an emitting source like the sun, the coherence time is about $10^{-15} \mathrm{sec}$ including interaction and Doppler effects. This magnitude, in terms of coherence length is approximately equal to few wave lengths. The coherence time can be up to $10^{-11} \mathrm{sec}$ for a discharge lamp (in terms of coherence length this is approximately equal to few millimetres). For a laser the coherence time grows up from $10^{-8}$ to $0.1 \mathrm{sec}$ (in terms of coherence length, this goes from $0.5 \mathrm{~m}$ up to $10^{7}$ metres approximately). The big differences among these values determine their different behaviour.

Now, if we consider the detector characteristics, we have to take account that the reaction time of the human eye is about $0.1 \mathrm{sec}$, for a photographic film, 0.001 or for a photomultiply, $10^{-9} \sec .(4)$.

In this way it is possible to specify the experimental quantitative conditions to get stable pattern of interference. For instance in Young experiments it is possible calculate: 
* when a source can be consider as a point comparing it with the other dimensions of the system,

* how critical is the monochromaticity of the light emitted by the sources, in terms of the length of coherence necessary to see a modulated pattern,

* what dimensions are important when it is necessary estimates the apartment of the slits and the wide of these,

* What happens when the light by both slits is polarized in the same plane or not?

It is possible to analyse the same kind of calculation in a Michelson Interferometer or in a system with polarisers and a retard plate.

As a conclusion, we considered that one most important achieves of this work is the integration of polarization phenomenum with interference and diffraction. The idea is to understand the different effects in terms of interference considering amplitude splitting, wavefront splitting, orthogonal components splitting and taking in account the monochromaticity of the source.

\section{5.- REFERENCES}

1 - Colombo de Cudmani Leonor et al, "Optica Física Básica. Estructurada alrededor del concepto de coherencia luminosa", Universidad Nacional de Tucumán, Publicación Nro. $1441,1990$.

2 - Colombo de Cudmani Leonor, Pesa Marta, Salinas Julia, "Presentación de un texto introductorio a la optica fisica, estructurado alrededor del concepto de coherencia luminosa", Didáctica de la Física y de la Química, Enseñanza de las Ciencias, Tomo 1, 1989

3 - Jaen Mirta - Colombo Elisa, "Cad. Cat. Ens. Fis.", Florianópolis, Brasil, v.8, nro.2, 144160,1991

4 - Crawford Frank S., Jr., "Ondas", Berkeley physics course - vol.3, Editorial Reverté, S.A., 1979

5 - Feynman Richard P., Lighton Robert B., Sands Matthew, "The Feynman Lectures on Physics", vol.I, Fondo Educativo Interamericano S.A., 1971 